\title{
Sistema Nacional de Redes Fito: uma abordagem sobre o desafio da produção de matérias primas vegetais com qualidade
}

\author{
National System of Phyto-Networks: an approach on the challenge of producing quality plant raw \\ materials
}

\author{
Julienny Dias dos Santos Araújo ${ }^{1 *}$, Moisés Teles Madureira ${ }^{1}$, Lorena Pitta Nascimento ${ }^{2}$, Marina \\ Constant Bruno Maciel $^{2}$
}

Como citar esse artigo. Araújo, JDS; Madureira, MT; Nascimento, LP; Maciel, MCB. Sistema Nacional de Redes Fito: uma abordagem sobre o desafio da produção de matérias primas vegetais com qualidade. Revista Teccen. 2021 Jul./Dez.; 14 (2): 17-27.

\section{Resumo}

A tradição sobre o conhecimento e o uso das plantas pelos povos de todo o mundo é uma prática reconhecida e incentivada pela Word Health Organization (WHO). Alinhado com as recomendações da WHO, o Brasil possui políticas públicas e programas que tem como um dos objetivos, promover a integração de produtores rurais à cadeia de produção e inovação de matérias primas oriundas da biodiversidade, fornecendo produtos de qualidade e a preços acessíveis no mercado. $\mathrm{O}$ presente trabalho está orientado para atender três objetivos: 1 - informar eventos importantes acerca do estabelecimento das bases do desenvolvimento tecnológico de produtos de origem vegetal no Brasil; 2 - abordar a área de controle da qualidade como parte inerente ao desenvolvimento tecnológico de "fito derivados"; 3 - desenvolver um planejamento de estimativa de custos, atendendo diretrizes especificadas pela WHO, para a implementação de um laboratório de controle de qualidade de matérias primas vegetais, a partir de premissas adotadas para uma unidade básica de produção. Constata-se que as RedesFito são uma realidade dentro do contexto brasileiro, onde as experiências dos participantes estão sendo integradas ao Sistema Nacional de Redes Fito, gerando conhecimento para o processo de inovação e visando a produção de produtos a partir da biodiversidade brasileira. Os custos estimados representam o resultado de uma primeira abordagem de planejamento, levando em consideração as diretrizes da WHO. Como conclusão, entende-se que: nível de complexibilidade tecnológica para o desenvolvimento de produtos da biodiversidade é grande, exigindo ainda a superação de limitações técnicas e de legislação; a implementação de um laboratório de controle de qualidade pode ser adiada num primeiro momento, já que a necessidade de garantir infraestrutura numa propriedade rural possa significar uma estratégia prioritária; a estimativa de custos pode ser revisada para valores abaixo dos obtidos neste primeiro planejamento na medida em que parcerias técnicas possam ser viabilizadas com participantes das redes que possuam condições de execução de parte ou todas as análises requeridas de controle de qualidade.

Palavras-Chave: Biodiversidade; Redes de inovação; Matéria-prima vegetal.

\begin{abstract}
The tradition of knowledge and use of plants by people all over the world is a practice recognized and encouraged by the Word Health Organization (WHO). Aligned with the WHO recommendations, Brazil has public policies and programs that have, as one of the objectives, to promote the integration of rural producers to the production and innovation chain of raw materials from biodiversity, providing quality products at affordable market prices. The present work is oriented to meet three objectives: 1 - to inform important events about the establishment of the bases of technological development of products of vegetable origin in Brazil; 2 - to approach the quality control area as an inherent part to the technological development of "phyto-derivatives"; 3 - to develop a cost estimate planning, meeting guidelines specified by WHO, for the implementation of a quality control laboratory of vegetable raw materials, from premises adopted for a basic production unit. It can be seen that the PhytoNetworks are a reality within the Brazilian context, where the experiences of the participants are being integrated into the National System of PhytoNetworks, generating knowledge for the innovation process and aiming at the production of products from the Brazilian biodiversity. The estimated costs represent the result of a first planning approach, taking into consideration the WHO guidelines. In conclusion, it is understood that: the level of technological complexity for the development of products from biodiversity is high, requiring the overcoming of technical and legislative limitations; the implementation of a quality control laboratory can be postponed for the first time, since the need to guarantee infrastructure in a rural property may mean a priority strategy; the cost estimate can be revised to values below those obtained in this first planning as technical partnerships can be made possible with network participants who are able to perform part or all of the analyses required for quality control.

Keywords: Biodiversity; Innovation Networks, Vegetable Raw Material.
\end{abstract}

Afiliação dos autores:

${ }^{1}$ Universidade de Vassouras, Vassouras, RJ, Brasil.

${ }^{2}$ Núcleo Ipê RedesFito Mata Atlântica,Vassouras, Rio de Janeiro, Brasil.

* Email para correspondência: araujojulienny@gmail.com 


\section{Introdução}

A World Heath Organization (2003) reconhece as práticas dos povos sobre o uso de plantas medicinais e estimula os países a empregarem esforços no sentido de desenvolverem políticas públicas que possam tornar tais práticas cada vez mais concretas, com produtos de qualidade disponíveis no mercado e com preços acessíveis aos usuários.

O Brasil é um país que detém uma tradição secular sobre o conhecimento e uso de plantas medicinais, influenciado em parte pelas contribuições originais das diversas etnias que compõem a população do país.

Por parte do Governo Federal, uma das grandes iniciativas que se teve registro foi a criação da Política Nacional de Plantas Medicinais e Fitoterápicos e do Programa Nacional de Plantas Medicinais e Fitoterápicos no ano de 2006, e que foram consolidadas em um único documento em 2016, vindo a ser chamado de Política e Programa Nacional de Plantas Medicinais e Fitoterápicos (PPNPMF).

Como um dos desdobramentos das ações do Governo Federal, a Fundação Oswaldo Cruz (Fiocruz), através da Portaria $\mathrm{n}^{\circ} 21$, de 30 de agosto de 2010 estabeleceu o Sistema Nacional de Redes de Inovação em Medicamentos da Biodiversidade (SNRF), ampliando as bases para a formação de Arranjos Eco produtivos Locais (AEPLs) por biomas.

Em seguida, os AEPLs (2020) se difundiram em núcleos de inovação, consolidando a RedesFito ao longo dos seis biomas nacionais brasileiros e estabelecendo formas de organização cooperativa entre diversos participantes, dentre os quais centros de pesquisa, populações tradicionais, empresas, associações da sociedade civil, universidades etc.

Criado em 2019 e sediado no Município de Vassouras/Rio de Janeiro, o Núcleo Ipê RedesFito é um dos polos pertencentes aos AEPLs da Rede Mata Atlântica, onde estão aportados alguns projetos de produção agrícola de alimentos e matérias primas vegetais, tais como óleos essenciais artesanais, buscando agregar conhecimento técnico e ajudar pequenos agricultores que vivem dessa prática a serem inseridos na atividade econômica da região (FIOCRUZ, 2019).

$\mathrm{O}$ presente trabalho tem como objetivo trazer dados informativos sobre as bases do desenvolvimento tecnológico de produtos de origem vegetal, balizado por uma metodologia que abrange as áreas mestras da biologia, da agronomia, da tecnologia química e farmacêutica para a produção de "fito derivados". Adicionalmente, o trabalho coloca em foco uma das preocupações mais relevantes do processo de desenvolvimento tecnológico que é a qualidade da matéria prima que venha a ser ofertada ao mercado. Finalizando, o trabalho faz um breve exercício de planejamento preliminar de custos envolvidos na implementação de um laboratório de controle de qualidade de matérias primas, acoplado à uma unidade de cultivo de plantas, como parte imprescindível da cadeia de produção, baseado nas recomendações feitas pela World Heath Organization (WHO).

\section{Metodologia}

As informações inseridas no presente trabalho, foram levantadas através de fontes documentais encontradas em plataformas da internet e em bibliografias impressas relacionadas ao tema.

Com a finalidade de proporcionar a melhor compreensão da estruturação do texto, através de uma leitura coerente com a boa organização, o escopo do presente trabalho está desdobrado conforme a evolução dos seguintes tópicos:

O tópico Desafio dos Fitoterápicos apresenta uma breve inserção do tema através da problemática relacionada à algumas barreiras técnicas a serem resolvidas no sentido de tornar a produção de matérias primas vegetais uma realidade consolidada no Brasil. O tópico Áreas Mestras do Desenvolvimento Tecnológico aborda as competências profissionais como um dos pontos de partida para o conhecimento dos processos inerentes à produção de um produto de origem vegetal. Com vistas ao desfecho de uma visão abrangente do tema, o tópico Controle da Qualidade de Matérias Primas Vegetais representa uma consequência das atividades contempladas nas áreas mestras, que remetem à preocupação com a procedência e a garantia da qualidade dos produtos que serão de alguma forma consumidos por usuários finais. Para tanto, torna-se oportuno e igualmente importantes um levantamento de custos para a implementação de infraestrutura de produção e de controle através da utilização de instrumentação e técnicas adequadas.

\section{O Desafio dos Fitoterápicos}

A cadeia de produção de um produto de origem vegetal, com vistas ao alcance de um produto medicamentoso deve envolver os processos necessários para garantir a procedência da matéria prima, desde a obtenção do material genético devidamente identificado até os processos de formulação do produto final, passando pelas etapas de processamento pós-colheita da planta e pelos processos de produção dos extratos, fechando o ciclo com a formulação do produto e realização de testes pré-clínicos e clínicos fases I, II e III (FIOCRUZ, 2004).

Siani (2003) apresenta uma visão da estrutura como uma forma de mapeamento de áreas mestras para o desenvolvimento tecnológico de produto de origem 
vegetal, objetivando um medicamento fitoterápico. As competências profissionais das áreas mestras se referem a:

- Botânica

- Agronomia

- Química / Enga Química

- Farmacêutica

- Biomédica e Médica

Cada uma das áreas mestras constituemse em desafios técnico-científicos, operacionais e organizacionais de cooperação entre diversos participantes para que toda a cadeia de desenvolvimento leve a um produto final fitoterápico, vencendo todas as barreiras interpostas pelas autoridades de saúde do país com relação a aprovação e registro de um produto terapêutico para uso humano.

Costa (2020) apresenta uma síntese dos eventos ao desenvolvimento tecnológico voltado para a produção de um fitoterápico, nos quais os principais participantes fazem parte de uma rede de cooperação, promovendo assim as trocas de experiências e conhecimentos que impulsionam o processo de inovação.

\section{Áreas Mestras do Desenvolvimento Tecnológico}

\section{Área Botânico/Agronômica}

Tendo como um dos pilares dessa área o acesso e a identificação da planta, bem como o cultivo e o manejo sustentável visando a produção e a distribuição de produtos com a qualidade requerida para consumo, o PPNPMF aponta diretrizes alinhadas com as recomendações da WHO.

O Ministério da Saúde (2016) ratificou as diretrizes do PPNPMF reconhecendo a necessidade de incentivar a fomentar associação de agentes da sociedade que ampliem a difusão da agricultura familiar e das agroindústrias de plantas medicinais.

Dentre as ações convergentes com as diretrizes do Ministério da Saúde verificam-se ações favoráveis, tais como a publicação de produções bibliográficas a respeito da produção agrícola, elaborada pela Empresa Brasileira de Pesquisa Agropecuária (EMBRAPA), abordando modernas técnicas dentro dos conceitos de produção orgânica e agroecológica.

Corrêa Junior (2013), apresenta um Manual Técnico de Boas Práticas de Produção Agrícola de Plantas Medicinais de uma forma ampla e generalista, que leva em consideração parâmetros de qualidade que devem ser objeto de atenção para a produção de plantas medicinais. Nesse sentido, tem-se uma visão organizacional de um empreendimento que vai desde o conhecimento da legislação pertinente ao tema até considerações sobre a comercialização do produto final. Os aspectos pertinentes à produção referem-se a:

- Identificação botânicas das espécies de plantas a serem produzidas

- Tecnologia de produção

- Manejo de mudas e sementes

- Qualidade do solo

- Sistema de irrigação

- Processos de colheita

- Beneficiamento primário da matéria prima

Todos os aspectos anteriormente citados dizem respeito ao que está dentro do espectro das áreas mestras Botânica e Agronômica, dando conta das etapas pertinentes à cadeia de desenvolvimento tecnológico.

\section{Área Química-Farmacêutica.}

Voltando à cadeia de desenvolvimento de fitoterápicos, a área mestra subsequente à Agronômica é a Química/Enga Química - Farmacêutica, que abarca as atividades desde o processamento pós-colheita até a formulação do produto. O escopo dessa parte do desenvolvimento tecnológico é por si bastante complexa e multidisciplinar, requerendo competências muito específicas para sua completa investigação e validação do fitoterápico que se tem interesse em comercializar.

O Instituto de Tecnologia em Fármacos (FarManguinhos) é uma das unidades da Fiocruz dedicada à Pesquisa, Desenvolvimento e Inovação em produtos fitoterápicos a partir de plantas brasileiras. Além dessa atribuição, o Instituto coordena os escritórios das redes de inovação em Fito (RedesFito) distribuídos pelos seis biomas nacionais, mantendo parcerias com diversas entidades, civis e oficiais visando a produção de produtos fitoterápicos.

Sharapin (2000), traz contribuições para a fundamentação de tecnologias que auxiliem no desenvolvimento da produção de fitoterápicos, estando consistente com as diretrizes internacionais preconizadas pela WHO.

Sinteticamente, o Quadro 1 esclarece as áreas necessárias para o processamento pós-colheita e seus respectivos objetivos, em consonância com as boas práticas preconizadas para a garantia da qualidade química e microbiológica das matérias-primas vegetais.

A partir da obtenção de extratos padronizados das plantas, que podem ainda ser levados ao nível de extratos secos padronizados, atinge-se um grau de valor agregado aos extratos que conferem aos mesmos maior estabilidade química e microbiológica, capazes de dar suporte aos estudos posteriores, que consistirão no desenvolvimento das formulações medicamentosas a serem submetidas aos ensaios pré-clínicos e clínicos. 
Quadro 1. Estrutura física da área de beneficiamento primário.

\begin{tabular}{|c|c|c|}
\hline Área & Processos & Objetivos \\
\hline \multirow[t]{3}{*}{$\begin{array}{l}\text { Benefi- } \\
\text { ciamento } \\
\text { primário }\end{array}$} & $\begin{array}{c}\text { Recebimento e separa- } \\
\text { ção de partes das plantas } \\
\text { (Processamento pós-co- } \\
\text { lheita) }\end{array}$ & $\begin{array}{l}\text { Manter a alta qualidade } \\
\text { da matéria prima, pre- } \\
\text { servando suas caracte- } \\
\text { rísticas físico-químicas } \\
\text { e evitando também a } \\
\text { degradação microbioló- } \\
\text { gica. }\end{array}$ \\
\hline & \multirow{2}{*}{$\begin{array}{l}\text { Lavagem (a depender da } \\
\text { parte usada da planta) }\end{array}$} & $\begin{array}{l}\text { Inspecionar visualmente } \\
\text { e remover partes conta- } \\
\text { minadas }\end{array}$ \\
\hline & & $\begin{array}{l}\text { Remover sujeira gros- } \\
\text { seira e realizar limpeza }\end{array}$ \\
\hline Secagem & Secagem das plantas & $\begin{array}{l}\text { Reduzir o teor de umi- } \\
\text { dade e conservar a esta- } \\
\text { bilidade química e mi- } \\
\text { crobiológica }\end{array}$ \\
\hline Moagem & Corte / Moagem & $\begin{array}{l}\text { Fracionar as partes da } \\
\text { planta para chegar a } \\
\text { uma apresentação física } \\
\text { na forma granulada e } \\
\text { facilitar o processo de } \\
\text { extração } 2\end{array}$ \\
\hline \multirow[t]{3}{*}{$\begin{array}{l}\text { Armaze- } \\
\text { namento }\end{array}$} & $\begin{array}{l}\text { Armazenamento (a de- } \\
\text { pender da parte usada da } \\
\text { planta) }\end{array}$ & $\begin{array}{c}\text { Estocar temporariamen- } \\
\text { te da matéria prima antes } \\
\text { de ser requisitada para o } \\
\text { processo de extração }\end{array}$ \\
\hline & & $\begin{array}{c}\text { óleos essenciais presen- } \\
\text { tes nas folhas frescas }\end{array}$ \\
\hline & $\begin{array}{c}\text { Extração } 2 \text { (Droga vege- } \\
\text { tal seca) }\end{array}$ & $\begin{array}{c}\text { Extrair por processo } \\
\text { adequado o material a } \\
\text { partir da planta seca }\end{array}$ \\
\hline $\begin{array}{l}\text { Controle } \\
\text { da Quali- } \\
\text { dade }\end{array}$ & $\begin{array}{l}\text { Análises físico-químicas } \\
\text { e microbiológicas dos } \\
\text { materiais ao longo do } \\
\text { processo produtivo }\end{array}$ & $\begin{array}{l}\text { Determinar perfil quí- } \\
\text { mico, rendimento, mas- } \\
\text { sa específica, teor de } \\
\text { cinzas, estabilidades e } \\
\text { demais parâmetros per- } \\
\text { tinentes à qualidade. }\end{array}$ \\
\hline
\end{tabular}

Fonte: adaptado de Sharapin (2000).

Em resumo, uma representação adaptada da visão elaborada por Siani (2003) é fornecida pelo fluxograma 1, mostrando a complexidade do desafio do desenvolvimento de um fitoproduto de origem vegetal como um processo composto por fases ao longo das áreas mestras.

\section{Controle da Qualidade de Matérias Primas Vegetais}

Admitindo-se que uma das melhores abordagens para enfrentar esse tipo de desafio deve ser, gradual e progressiva, em iniciativas que poderiam nascer em parte da cadeia como um todo, porém com a necessidade de se interligar com as outras partes a fim de resolver eventuais gargalos.

Uma parte do processo que não pode estar descolada do processo de produção de um produto de origem vegetal qualificado é a inspeção e o controle da qualidade. Certamente, ele é uma parte integrante de toda a cadeia de produção, desde a área botânicoagronômica até os estudos clínicos.

No que se refere ao controle da qualidade da matéria prima como droga vegetal, a qual se trata dos processos posteriores à colheita da planta, as recomendações são bastante específicas sobre os cuidados com a manipulação e os ensaios físicoquímicos e microbiológicos que são preconizados pela WHO.

WHO (2011) define uma coletânea de testes a serem feitos em matérias primas vegetais com uma forma de dar suporte no desenvolvimento de padrões de qualidade nessa área, complementados pela legislação e por normas regulatórias locais de cada país. Nesse sentido, a determinação de materiais estranhos, da granulometria, da água e matérias voláteis, e de matérias extraíveis, determinação das cinzas, dos óleos essenciais e dos taninos, determinação de índice de espuma, de resíduos de pesticidas, de micro-organismos e de aflatoxinas, o exame macroscópico e microscópico e a cromatografia de camada fina, são os principais

Fluxograma 1. Fases do Desenvolvimento Tecnológico de um Fitoterápico (Macroprocessos).

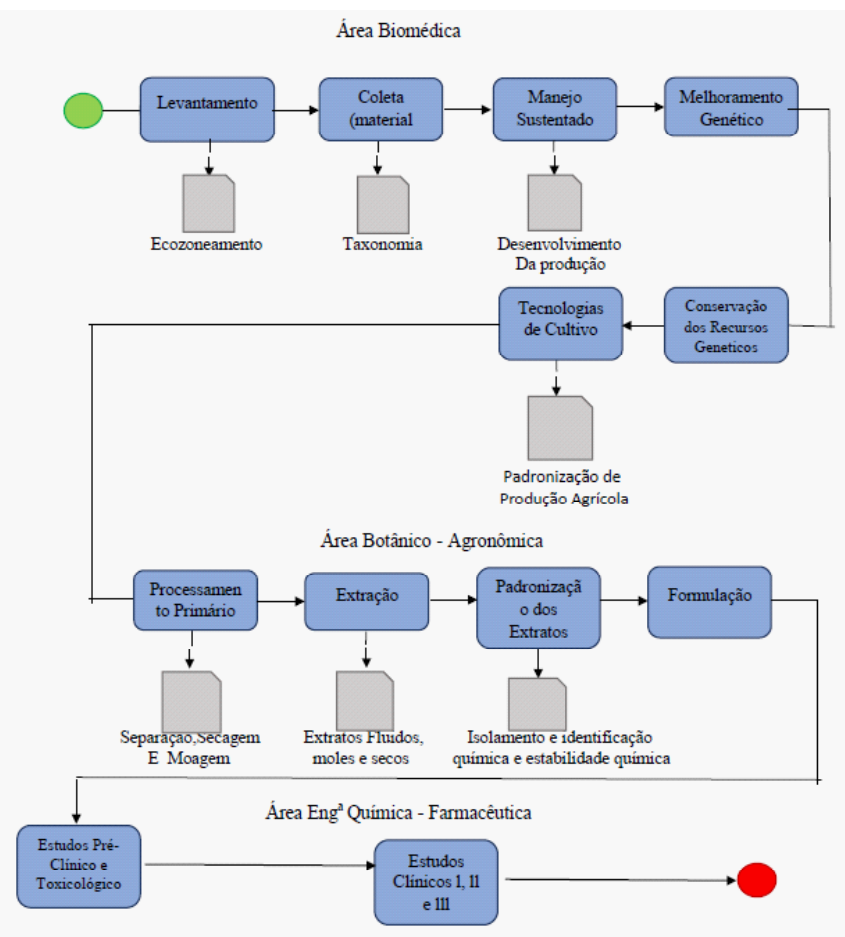

Fonte: Adaptado de Siani (2003). 
parâmetros de qualidade a serem controlados.

Segundo as recomendações da $\mathrm{WHO}$, as partes da planta (raiz, caule, galhos, folhas, flores e/ou partes aplicáveis conforme a natureza da planta) devem ser cuidadosamente separadas quando do recebimento para o processamento primário (após a colheita) e tratadas conforme os ensaios que estejam previstos.

Uma experiência atual, encontrada no curso desta pesquisa, que está mais próxima do modelo apresentado na Fluxograma 1 encontra em andamento na unidade de Far-Manguinhos.

APlataformaAgroecológicadeFitomedicamentos (PAF) é patrocinada pela Fiocruz, e se dedicada à padronização de produtos da biodiversidade através da gestão de vários laboratórios integrados à visão do desenvolvimento tecnológico de um fitoterápico (PAF, 2021)

Como forma de estimular maior envolvimento de outros agentes e disseminação de discussões sobre o tópico da "qualidade", que é de fundamental importância para assegurar a procedência da matéria prima, foi elaborado um exercício de planejamento preliminar, seguindo as recomendações da WHO, no intuito de estimar os custos envolvidos na implementação de um projeto estruturante de uma unidade de produção de matérias primas.

Para tanto, o planejamento levou em consideração a marcação de algumas premissas como bases da estimativa, que consistiram em:

- Definição da área de produção agrícola em $10.000 \mathrm{~m}^{2}$ (1 hectare), a ser considerada como uma unidade básica;

- Estabelecimento de uma produção anual de 2 toneladas de planta fresca;

- Planejamento de materiais de consumo para o início da produção e suprimento por um ano.

O Quadro 2 mostra a resultado obtido a partir das premissas assumidas, em termos de custos estimados para implementação de infraestrutura física.

O Quadro 3 mostra a resultado obtido a partir das premissas assumidas, em termos de custos estimados para implementação da área de produção agrícola.

Quadro 2. Estimativa de custos para implementação da infraestrutura física.

\begin{tabular}{|c|c|c|c|c|c|}
\hline \multirow{2}{*}{\multicolumn{4}{|c|}{ PLANILHA DE CUSTOS (ESTIMATIVA) }} & Emissão & REVISÃO \\
\hline & & & & outubro, 2021 & 0 \\
\hline \multicolumn{4}{|c|}{ IDENTIFICAÇÃO } & \multirow{2}{*}{$\begin{array}{l}\text { Preço Unitário } \\
\text { (R\$) }\end{array}$} & \multirow{2}{*}{$\begin{array}{l}\text { Preço Total } \\
\text { (R\$) }\end{array}$} \\
\hline UNIDADE DE PRODUÇÃO & DESCRIÇÃO & QUANT & UND. & & \\
\hline \multicolumn{4}{|l|}{ Infraestrutura física } & & \\
\hline $\begin{array}{c}\text { Prospecção e construção de poço } \\
\text { artesiano }\end{array}$ & $\begin{array}{l}\text { Complementar a rede de abastecimen- } \\
\text { to de água para atender as áreas }\end{array}$ & - & - & $20.000,00$ & $20.000,00$ \\
\hline Análise da água & $\begin{array}{l}\text { Assegurar condições de uso da água } \\
\text { em níveis adequados de qualidade }\end{array}$ & - & - & 500,00 & 500,00 \\
\hline Instalação de Cisterna $\left(30 \mathrm{~m}^{3}\right)$ & $\begin{array}{l}\text { Armazenar água para suprir sistema } \\
\text { de irrigação e outros serviços }\end{array}$ & - & - & $2.000,00$ & $2.000,00$ \\
\hline Sistema de Irrigação & $\begin{array}{l}\text { Disponibilizar água para o processo } \\
\text { de cultivo }\end{array}$ & - & - & $10.000,00$ & $10.000,00$ \\
\hline $\begin{array}{l}\text { Adequação / reforma galpão de } \\
\text { agrícola }\left(60 \mathrm{~m}^{2}\right)\end{array}$ & $\begin{array}{l}\text { Manter e gerenciar materiais e } \\
\text { equipamentos agrícolas }\end{array}$ & 60 & $\mathrm{~m}^{2}$ & $12.000,00$ & $12.000,00$ \\
\hline $\begin{array}{l}\text { Preparação da área de matrizes } \\
\text { de mudas }\left(5.000 \mathrm{~m}^{2}\right)\end{array}$ & $\begin{array}{l}\text { Prover área suficiente para receber e } \\
\text { cultivar mudas /sementes }\end{array}$ & 200 & $\mathrm{~m}^{2}$ & $3.000,00$ & $3.000,00$ \\
\hline $\begin{array}{l}\text { Preparação da área de plantio } \\
\left(10.000 \mathrm{~m}^{2}\right)\end{array}$ & $\begin{array}{l}\text { Analisar solo e realizar a correção } \\
\text { para posterior plantio }\end{array}$ & 10.000 & $\mathrm{~m}^{2}$ & $5.000,00$ & $5.000,00$ \\
\hline $\begin{array}{l}\text { Adequação de instalações } \\
\text { (elétrica, hidráulica) }\end{array}$ & $\begin{array}{l}\text { Disponibilizar cargas necessárias } \\
\text { ao funcionamento de máquinas e } \\
\text { equipamentos }\end{array}$ & - & - & $2.000,00$ & $2.000,00$ \\
\hline $\begin{array}{l}\text { Adequação da área de } \\
\text { processamento - laboratório }\end{array}$ & $\begin{array}{l}\text { Atender espaço necessário para } \\
\text { processar extratos e realizar controle } \\
\text { da qualidade }\end{array}$ & 100 & $\mathrm{~m}^{2}$ & $15.000,00$ & $15.000,00$ \\
\hline
\end{tabular}

Fonte: Autores, 2021. 
Quadro 3. Custos estimados para implementação da área de produção agrícola.

\begin{tabular}{|c|c|c|c|c|c|}
\hline \multirow{2}{*}{\multicolumn{4}{|c|}{ PLANILHA DE CUSTOS (ESTIMATIVA) }} & Emissão & REVISÃO \\
\hline & & & & outubro, 2021 & 0 \\
\hline \multicolumn{4}{|c|}{ IDENTIFICAÇÃO } & \multirow{2}{*}{$\begin{array}{l}\text { Preço Unitário } \\
\text { (R\$) }\end{array}$} & \multirow[b]{2}{*}{$\begin{array}{l}\text { Preço Total } \\
\text { (R\$) }\end{array}$} \\
\hline UNIDADE DE PRODUÇÃO & DESCRIÇÃO & QUANT & UND. & & \\
\hline \multicolumn{6}{|l|}{ Área de produção agrícola } \\
\hline Enxada & \multirow{29}{*}{$\begin{array}{l}\text { Instrumentos de trabalho de campo na } \\
\text { condução das rotinas do dia a dia }\end{array}$} & 10 & peça & 36,52 & 365,20 \\
\hline Enxadinha & & 10 & peça & 29,48 & 294,80 \\
\hline Tesoura poda & & 4 & peça & 29,90 & 119,60 \\
\hline Vassoura de aço para grama/capim & & 4 & peça & 47,90 & 191,60 \\
\hline Ancinho & & 8 & peça & 35,40 & 283,20 \\
\hline Carrinho de Mão & & 6 & peça & 231,95 & $1.391,70$ \\
\hline Tesoura de cortar grama/capim & & 5 & peça & 39,80 & 199,00 \\
\hline Balde de plástico & & 5 & peça & 21,90 & 109,50 \\
\hline Cavadeira & & 3 & peça & 68,21 & 204,63 \\
\hline Pá & & 4 & peça & 35,25 & 141,00 \\
\hline Enxadão & & 6 & peça & 59,25 & 355,50 \\
\hline Roçadeira costal & & 2 & peça & 849,00 & $1.689,00$ \\
\hline Tubete para mudas & & 5.000 & peça & 0,78 & $3.900,00$ \\
\hline Tridente & & 4 & peça & 155,71 & 622,84 \\
\hline Machado & & 2 & peça & 46,71 & 93,42 \\
\hline Peneira & & 10 & peça & 24,16 & 241,60 \\
\hline Lima & & 3 & peça & 33,23 & 99,69 \\
\hline Arado & & 1 & peça & $5.000,00$ & $5.000,00$ \\
\hline Canivete & & 8 & peça & 24,90 & 199,20 \\
\hline Trena $(100 \mathrm{~m})$ & & 2 & peça & 45,90 & 91,80 \\
\hline Fio de nylon & & 5 & peça & 11,72 & 58,60 \\
\hline Pazinha de jardineiro & & 8 & peça & 16,00 & 128,00 \\
\hline Podão & & 1 & peça & 300,00 & 300,00 \\
\hline Serrote de poda & & 2 & peça & 70,00 & 140,00 \\
\hline Foice & & 5 & peça & 60,00 & 300,00 \\
\hline Facão & & 4 & peça & 50,00 & 200,00 \\
\hline Machadinha & & 3 & peça & 30,00 & 90,00 \\
\hline Esmeril & & 1 & peça & 150,00 & 150,00 \\
\hline Torno médio & & 1 & peça & 100,00 & 100,00 \\
\hline Triturador de galhos & $\begin{array}{l}\text { Triturar material para realização } \\
\text { compostagens e outras atividades }\end{array}$ & 1 & peça & $4.000,00$ & $4.000,00$ \\
\hline Micro trator & $\begin{array}{c}\text { Dar suporte às tarefas de cultivo das } \\
\text { espécies }\end{array}$ & 1 & und. & $5.000,00$ & $5.000,00$ \\
\hline Balança $100 \mathrm{~kg}$ & $\begin{array}{c}\text { Determinar peso da biomassa gerada } \\
\text { no campo }\end{array}$ & 1 & peça & $3.500,00$ & $3.500,00$ \\
\hline
\end{tabular}


O Quadro 4 apresenta custos estimados para implementação das áreas de beneficiamento e de controle da qualidade.
O Quadro 5 mostra a resultado obtido a partir das premissas assumidas, em termos de custos estimados para implementação de escritório, materiais de consumo,

Quadro 4. Custos estimados das áreas de beneficiamento e controle da qualidade.

\begin{tabular}{|c|c|c|c|c|c|}
\hline \multirow{2}{*}{\multicolumn{4}{|c|}{ PLANILHA DE CUSTOS (ESTIMATIVA) }} & Emissão & REVISÃO \\
\hline & & & & outubro, 2021 & 0 \\
\hline \multicolumn{4}{|c|}{ IDENTIFICAÇÃO } & \multirow{2}{*}{$\begin{array}{l}\text { Preço Unitário } \\
\text { (R\$) }\end{array}$} & \multirow{2}{*}{$\begin{array}{l}\text { Preço Tota } \\
\text { (R\$) }\end{array}$} \\
\hline UNIDADE DE PRODUÇÃO & DESCRIÇÃO & QUANT & UND. & & \\
\hline \multicolumn{6}{|l|}{$\begin{array}{l}\text { Área de Processamento } \\
\text { (Beneficiamento/Extração) }\end{array}$} \\
\hline $\begin{array}{c}\text { Tanque de Extrator Aço Inox (50 } \\
\text { litros) }\end{array}$ & $\begin{array}{l}\text { Extrair componentes de droga vegetal } \\
\text { seca }\end{array}$ & 1 & und. & $8.000,00$ & $8.000,00$ \\
\hline Balança Semi industrial (100 kg) & $\begin{array}{l}\text { Determinar peso e rendimento de } \\
\text { material pós-colheita }\end{array}$ & 1 & und. & $3.500,00$ & $3.500,00$ \\
\hline $\begin{array}{c}\text { Extrator por Arraste a vapor (10 } \\
\mathrm{kg})\end{array}$ & Extrair óleos essenciais & 1 & unid. & $12.000,00$ & $12.000,00$ \\
\hline $\begin{array}{c}\text { Câmara de secagem de planta } \\
\text { fresca }\end{array}$ & $\begin{array}{l}\text { Remover teor de água de partes das } \\
\text { plantas }\end{array}$ & 1 & unid. & $10.000,00$ & $10.000,00$ \\
\hline Moinho de facas aço inox & $\begin{array}{l}\text { Triturar e reduzir tamanho do material } \\
\text { a ser extraído }\end{array}$ & 1 & unid. & $6.000,00$ & $6.000,00$ \\
\hline $\begin{array}{l}\text { Vidraria geral, materiais acessórios } \\
\text { e de consumo }\end{array}$ & $\begin{array}{c}\text { Gerir suporte às atividades de rotina } \\
\text { da área }\end{array}$ & - & Geral & $2.000,00$ & $2.000,00$ \\
\hline Evaporador rotativo & $\begin{array}{l}\text { Recuperar solvente dos extratos } \\
\text { fluidos }\end{array}$ & 1 & unid. & $1.500,00$ & $1.500,00$ \\
\hline Bomba de vácuo & $\begin{array}{l}\text { Auxiliar o processo de recuperação de } \\
\text { solvente no evaporador }\end{array}$ & 1 & unid. & $1.000,00$ & $1.000,00$ \\
\hline \multicolumn{6}{|l|}{$\begin{array}{c}\text { Área de Controle da Qualidade } \\
\text { (Laboratório) }\end{array}$} \\
\hline Termômetro de bulbo de mercúrio & \multirow{9}{*}{$\begin{array}{c}\text { Equipar a área para conduzir os } \\
\text { trabalhos das rotinas do dia a dia do } \\
\text { laboratório }\end{array}$} & 3 & unid. & 44,90 & 134,70 \\
\hline Lupa & & 3 & unid. & 26,50 & 79,50 \\
\hline Vidraria geral & & - & Geral & $1.000,00$ & $1.000,00$ \\
\hline Reagentes & & - & Geral & $3.000,00$ & $3.000,00$ \\
\hline Geladeira (570 litros) & & 2 & unid. & $6.000,00$ & $12.000,00$ \\
\hline Freezer comercial (420 litros) & & 1 & unid. & $3.000,00$ & $3.000,00$ \\
\hline Forno Mufla & & 1 & unid. & $4.000,00$ & $4.000,00$ \\
\hline Deionizador de água & & 1 & unid. & $2.500,00$ & $2.500,00$ \\
\hline Capela de exaustão & & 1 & unid. & $5.000,00$ & $5.000,00$ \\
\hline Densímetro & \multirow{5}{*}{$\begin{array}{l}\text { Realizar ensaios físico-químicos em } \\
\text { amostras no laboratório }\end{array}$} & 1 & unid. & $3.000,00$ & $3.000,00$ \\
\hline Balança analítica & & 1 & unid. & $2.000,00$ & $2.000,00$ \\
\hline Balança semi-analítica & & 1 & unid. & $1.500,00$ & $1.500,00$ \\
\hline $\begin{array}{l}\text { Kit de cromatografia de camada } \\
\text { fina }\end{array}$ & & 1 & Geral & $10.000,00$ & $10.000,00$ \\
\hline Medidor de $\mathrm{pH}$ & & - & unid. & $1.600,00$ & $1.600,00$ \\
\hline Microscópio & \multirow{4}{*}{$\begin{array}{l}\text { Realizar ensaios microbiológicos em } \\
\text { amostras no laboratório }\end{array}$} & 1 & unid. & $4.000,00$ & $4.000,00$ \\
\hline Contador de colônia & & 1 & unid. & $1.000,00$ & $1.000,00$ \\
\hline Estufa de cultura & & 1 & unid. & $2.000,00$ & $2.000,00$ \\
\hline Fluxo laminar & & 1 & unid. & $10.000,00$ & $10.000,00$ \\
\hline
\end{tabular}


Quadro 5. Custos estimados da área de escritório e materiais de consumo.

\begin{tabular}{|c|c|c|c|c|c|}
\hline \multirow{2}{*}{\multicolumn{4}{|c|}{ PLANILHA DE CUSTOS (ESTIMATIVA) }} & Emissão & REVISÃO \\
\hline & & & & outubro, 2021 & 0 \\
\hline \multicolumn{4}{|c|}{ IDENTIFICAÇÃO } & \multirow{2}{*}{$\begin{array}{l}\text { Preço Unitário } \\
\text { (R\$) }\end{array}$} & \multirow{2}{*}{$\begin{array}{l}\text { Preço Tota } \\
\text { (RS) }\end{array}$} \\
\hline UNIDADE DE PRODUÇÃO & DESCRIÇÃO & QUANT & UND. & & \\
\hline \multicolumn{6}{|l|}{ Escritório } \\
\hline Notebook & \multirow{4}{*}{$\begin{array}{l}\text { Dar suporte às atividades } \\
\text { administrativas da unidade de } \\
\text { produção }\end{array}$} & 2 & unid. & $3.000,00$ & $6.000,00$ \\
\hline Impressora & & 1 & unid. & $1.111,00$ & $1.111,00$ \\
\hline GPS & & 1 & unid. & $3.000,00$ & $3.000,00$ \\
\hline $\begin{array}{l}\text { Mobiliário de escritório (cadeira, } \\
\text { mesa, armário, estante etc) }\end{array}$ & & - & Geral & $5.000,00$ & $5.000,00$ \\
\hline \multicolumn{6}{|l|}{$\begin{array}{c}\text { Equipamento de proteção (EPI) - } \\
\text { Área agrícola } \\
\end{array}$} \\
\hline Luva de jardinagem & \multirow{11}{*}{$\begin{array}{l}\text { Proporcionar segurança durante o } \\
\text { desempenho eventual ou de rotina das } \\
\text { atividades dos trabalhadores }\end{array}$} & 12 & par & 1,99 & 23,88 \\
\hline Bota galocha & & 12 & par & 57,90 & 694,80 \\
\hline Avental azul com bolso & & 15 & peça & 42,17 & 632,55 \\
\hline Óculos proteção & & 10 & peça & 19,95 & 199,50 \\
\hline Luva descartável & & 12 & par & 2,75 & 33,00 \\
\hline Viseira protetor facial & & 6 & peça & 7,00 & 42,00 \\
\hline Caneleira & & 6 & par & 23,00 & 138,00 \\
\hline Luva de couro & & 6 & par & 150,00 & 900,00 \\
\hline Capa de chuva & & 10 & peça & 27,00 & 270,00 \\
\hline Bota de chuva & & 6 & par & 100,00 & 600,00 \\
\hline Protetor auricular abafador & & 10 & peça & 45,00 & 450,00 \\
\hline \multicolumn{6}{|l|}{$\begin{array}{l}\text { Equipamento de proteção (EPI) - } \\
\text { Laboratório }\end{array}$} \\
\hline Óculos de proteção & \multirow{3}{*}{$\begin{array}{l}\text { Proporcionar segurança durante o } \\
\text { desempenho eventual ou de rotina das } \\
\text { atividades dos trabalhadores }\end{array}$} & 12 & peça & 190,00 & $2.280,00$ \\
\hline Guarda pó & & 20 & peça & 70,00 & $1.400,00$ \\
\hline Touca descartável & & 500 & peça & 0,24 & 120,00 \\
\hline & & & & \multicolumn{2}{|c|}{ TOTAL FINAL (R\$) } \\
\hline & & & & \multicolumn{2}{|c|}{$231.777,81$} \\
\hline
\end{tabular}

Fonte: Autores, 2021.

fechando o levantamento total.

Um levantamento feito no mês de outubro de 2021 estimou o valor total dos custos em $\mathrm{R} \$ 231.777,81$, que se referem mais especificamente a aquisição de:

- Serviços de adequação das áreas básicas necessárias ao funcionamento;

- Materiais de consumo para um ano de operação

- Equipamentos permanentes;

- Equipamentos de proteção individual.

Uma configuração física das áreas planejadas pode ser representada pela Figura 1, a fim de proporcionar uma visão panorâmica da produção. 


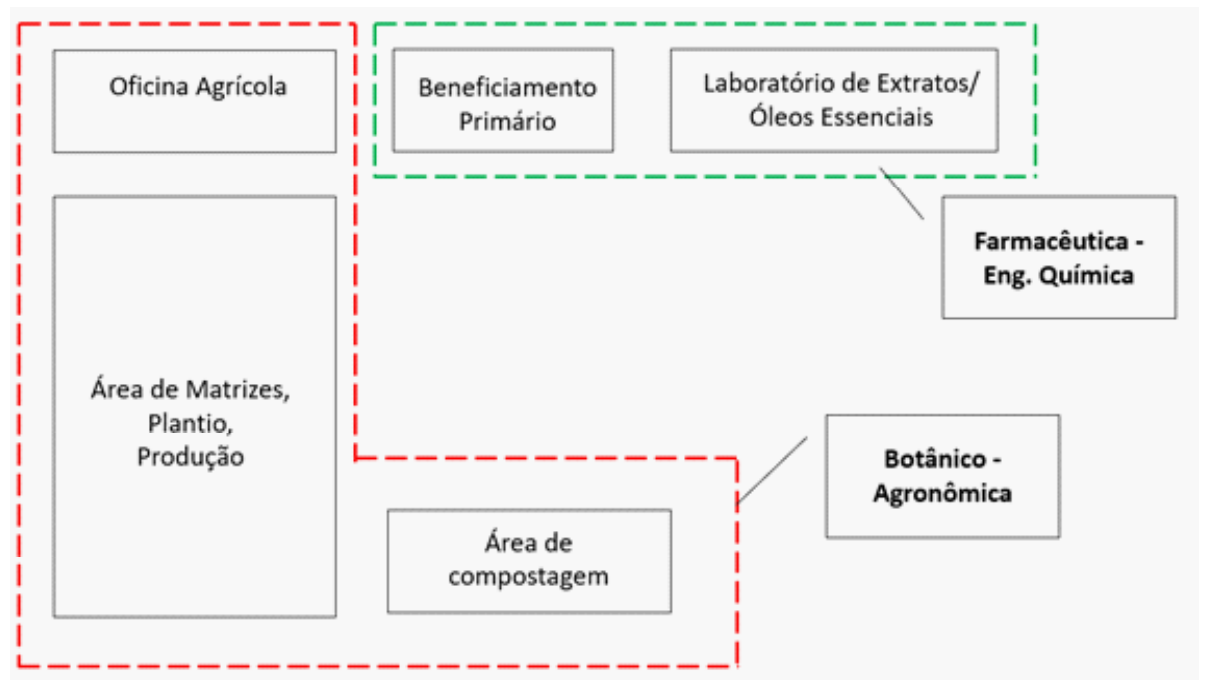

Figura 1. Desenho esquemático da unidade produtiva.

Fonte: Autores, 2021.

\section{Resultados}

Com a coleta de informações através deste trabalho, os resultados mostram uma evolução importante quanto as articulações entre os vários participantes da cadeia de desenvolvimento tecnológico de "fito", demonstrada pela formação e consolidação de redes de inovação com o passar do tempo. Atualmente, as RedesFito são uma realidade dentro do contexto brasileiro, onde são trocadas informações oriundas dos participantes que atuam nas próprias redes, servindo de base para a construção do conhecimento científico e do desenvolvimento tecnológico nos biomas.

No que se refere à questão voltada para a qualidade das matérias primas e produtos que venham a ser oriundos de espécies de plantas, verificou-se um nível de exigência em termos técnicos bastante elevado, considerando as diretrizes da $\mathrm{WHO}$ e o fato de que o alvo do processo de desenvolvimento é um medicamento de origem vegetal, portanto tendo que cumprir rigorosos critérios de aprovação. Adicionalmente, destaca-se que o escopo desta pesquisa foi limitado pela etapa de processamento pós-colheita da planta (beneficiamento primário e produção de extratos).

Os custos estimados, embora preliminares, resultaram num montante da ordem de centenas de milhares de reais necessários ao investimento. Os preços dos itens foram consultados em portais de fornecedores, disponíveis na internet e em consultas diretas realizadas em estabelecimentos comerciais da região de Vassouras/ Rio de Janeiro.

\section{Discussão}

As recomendações feitas pela $\mathrm{WHO}$ orientam os processos para uma produção com alto grau de qualidade desde as etapas produção agrícola até os processos de extração. Essas etapas satisfazem regras internacionais vigentes, agregando maior valor ao produto final. $\mathrm{O}$ alinhamento com as regras internacionais de produção e controle pode vir a ser uma vantagem bastante interessante, na medida em que o processo de exportação da matéria prima venha se tornar um objetivo do produtor.

A obtenção de óleos essenciais é um dos interesses ligados à produção de matérias primas vegetais, devido às suas funcionalidades como medicamentos, cosméticas e outras. A extração de óleos essenciais por arraste vapor é um processo de baixo custo de implementação e operação, sendo assim um processamento favorável para a implementação em áreas de baixa renda, já que para a produção não precisa de grandes conhecimentos teóricos e técnicos para serem operados, facilitando assim a produção em grande escala e baixo custo de operação, o que se tornaria viável através de programas de incentivo e financiamento público de projetos.

No entanto, os custos estimados nesta pesquisa podem contrastar com a realidade social e econômica de muitos produtores rurais, sem falar também da questão da formação técnica. Nesse contexto, não está fora de questão a possibilidade de que as condições reais dos produtores careçam de infraestrutura física adequada, tal como coleta e tratamento de esgotos, acesso à água em boas condições de consumo, regularização da propriedade rural, conhecimento de técnicas de produção orgânica e/ou agroecológica etc.

Segundo dados do último censo do IBGE (2010), cerca de $15,28 \%$ dos brasileiros vivem na zona rural, no estado do Rio de Janeiro cerca de 525.690 pessoas 
fazem parte dessa estimativa, a grande maioria dessas pessoas vivem com a ajuda de programas do governo para auxiliar na renda que possuem, já que a maioria não consegue receber por mês um salário-mínimo ou até mesmo não tem nenhuma fonte de renda. Por esse motivo, a implementação de programas em que essas pessoas retornam aos estudos e já são inseridas no mercado de trabalho gera para ambas as partes muitos fatores favoráveis.

A Associação das Mulheres Agricultoras e Produtoras Artesanais das Regiões Sul e Centro Sul Fluminense - AMARSUL com base nas necessidades da região visa produzir e comercializar óleos essenciais de forma artesanal, visando o emprego desse produto em variadas finalidades: proporcionar sabor e aroma aos alimentos, aplicação em bebidas, além dos usos terapêuticos por suas propriedades multifuncionais, participando de todas as etapas para a produção da mesma, desde a preparação do solo para o cultivo da matéria prima até sua comercialização. A proposta contempla a manutenção da Unidade Produtiva de Plantas Medicinais localizada em Demétrio Ribeiro, Distrito de Vassouras, RJ, suprida com mudas de plantas medicinais quimiotipadas, provindas das Unidades de Produção da Fiocruz. A espécie inicialmente cultivada é o Cymbopogon citratus, conhecida popularmente como capim-limão. Cultivam-se outras espécies, utilizadas no dia a dia das associadas, oriundas das suas hortas.

A implementação de projetos estruturantes que contemplem pequenos produtores rurais pode ser mais bem sucedida através de uma estratégia de abordagem que, em primeiro lugar, transforme a realidade local das pessoas, através de ações que promovam um diagnóstico situacional da área de implantação do projeto, a implementação de sistema de saneamento básico rural, um estímulo a formação de cooperativas, palestras e reuniões motivacionais, assistência técnica para capacitação de produtores, melhoria da formação acadêmica e qualificação técnica e Integração à programas de agricultura familiar.

Como uma forma de evolução do processo de desenvolvimento do produtor, o próximo passo desse processo evolutivo poderia ser a introdução de novos projetos, tais o plantio e produção de plantas sob regras mais restritas de qualidade e controle, tais como as medicinais.

\section{Considerações finais}

Os macroprocessos da cadeia do desenvolvimento tecnológico de fitoterápicos (exibida pelo fluxograma 1) permitem concluir que o nível de complexidade envolvido é muito grande. Até que se possa atingir um processo de desenvolvimento próprio e suficientemente consistente, o sistema nacional de redes deve vencer barreiras técnicas e de legislação. Apenas para citar como um exemplo, a etapa de eco zoneamento, que está ligada ao acesso ao material genético já é um ponto que precisa estar bem esclarecido.

Uma das propostas das RedesFito é a do desenvolvimento por biomas, que estão espalhados pelo território brasileiro, e contam com diversos agentes participantes. Dentre os agentes estão os produtores rurais, que como uma das pontas de produção de matérias primas que necessitarão de recursos financeiros e tecnológicos para se consolidarem como fontes de produção sustentáveis e permanentes. Experiências de parcerias entre indústrias e produtores rurais já são observadas no país. Logo, a tendência é de que novos modelos de parcerias possam se multiplicar.

Os custos estimados nesta pesquisa podem ainda ser reduzidos na medida em que as universidades, como um dos agentes participantes do processo, possam contribuir com a contrapartida de realização de análises das matérias primas produtos, uma vez que equipadas com laboratórios adequados, contam com a qualificação de seus quadros técnicos, que podem prestar os serviços de análises.

\section{Referências}

Agyarko, E., Akunyili, D. N., Alkofahi, A. S., Anderson, L., Aung, U. M. K., Boukef, K., ... Shakhnoz, A. (2003). WHO guidelines on good agricultural and collection practices (GACP) for medicinal plants. Recuperado de: https:// apps.who.int/iris/bitstream/handle/10665/42783/9241546271.pdf

Azevedo, C. D. de., \& Moura, M. A. de., (2010). Cultivo de plantas medicinais: guia prático. [Manual Técnico]. Niterói: Programa Rio Rural 2010

Corrêa, C., Jr. \& Scheffer, M. C. (2013). Boas Práticas Agrícolas (BPA) de Plantas Medicinais, Aromáticas e Condimentares. Recuperado de: https:// www.fitoterapiabrasil.com.br/sites/default/files/documentos-oficiais/bpaplantas-medicinais-aromaticas-condimentares-ok.pdf

Costa, A. C. S. dos, Rezende, K. S., Faustino, F. F., Maciel, M. C. B., Madureira, M. T. (2020) Desenvolvimento tecnológico em produtos da biodiversidade brasileira através das Redes de Inovação em fito: Uma referência ao conceito de Arranjos Produtivos Locais e a experiência de um Núcleo de Inovação aplicada no município de Vassouras - RJ. Programa de Mestrado Profissional em Ciências Ambientais. (pp. 468-497). Universidade de Vassouras, 2020. ISBN: 978-65-87918-11-2. Recuperado de: http:// editora.universidadedevassouras.edu.br/index.php/PT/issue/view/197.

Fiocruz., (n.d.). RedesFito, Inovação de Medicamentos da Biodiversidade Recuperado de: RedesFito - AEPLs (fiocruz.br)

Instituto Brasileiro Geografia e Estatística- IBGE. (2010). População brasileira., Recuperado de: https://censo2010.ibge.gov.br/sinopse/index. php?dados $=8$

Koketsu, M., \& Gonçalves, S. L., (1991) Óleos essências e sua extração por arrase a vapor. EMBRAPA CTAA, 24p. Rio de Janeiro: Rio de Janeiro.

Lourenço, J. A. A., (2017). DESTILAÇÃO INDUSTRIAL DE ÓLEOS ESSENCIAIS. In A. C. Figueiredo, J. G. Barroso, L. G Pedro (Eds.) Potencialidades e Aplicações das Plantas Aromáticas e Medicinais. (pp. 8095). Portugal: Lisboa.

Mercado Livre., (2021). Recuperado de: https://www.mercadolivre.com.br

Ministério da Saúde. (2016). Política e Programa Nacional de Plantas Medicinais e Fitoterápicos. Recuperado de: https://bvsms.saude.gov.br/bvs/ publicacoes/politica_programa_nacional_plantas_medicinais_fitoterapicos. 
pdf

MODERNIZAÇ̃̃O DAS ESTATÍSTICAS ECONÔMICAS. (n.d.) Apresentado no CONGRESSO NACIONAL DE PESQUISA E ENSINO EM CIÊNCIAS - CONAPESC. Paraíba: Campina Grande.Secretaria Municipal de Saúde e Defesa Civil - Rio de Janeiro., (2012). Manual de Cultivo de Plantas Medicinais. Subgerência do Programa de Plantas Medicinais e Fitoterapia. Gerencia do Programa de Práticas Integrativas e Complementares., [Série B. Normas e Manuais Técnicos]., Rio de Janeiro: Rio de Janeiro.

Sharapin, N. (2000). Fundamentos de Tecnologia de Produtos Fitoterápicos. In Pinzón, R. S., (Ed). Colômbia: Santafé de Bogotá.

Silva, L. N., Santos, S. B. F., Paixão, I. C. S., Vilela, R. C. C., \& Pereira, T. S. dos., (n.d.) INDUSTRIA DE ÓLEOS ESSENCIAIS NO BRASIL: UMA PERSPECTIVA A PARTIR DO PROGRAMA DE MODERNIZAÇÃO DAS ESTATÍSTICAS ECONÔMICAS. Apresentado no CONGRESSO NACIONAL DE PESQUISA E ENSINO EM CIÊNCIAS - CONAPESC. Paraíba: Campina Grande.

World Health Organization. (1998). Quality control methods for medicinal plant materials. Recuperado de: https://books.google.com.br/books?hl=pt$\mathrm{BR} \& 1 \mathrm{r}=\& \mathrm{id}=$ ZjizDwAAQBAJ\&o $=$ =fnd $\& p \mathrm{p}=\mathrm{PT} 3 \& \mathrm{dq}=$ Quality + contro $1+$ methods + for + medicinal + plant + materials, \&ots $=y \operatorname{RsVCfaNBg} \&$ sig $=$ EQ1N7sQK-7BfnqFnqph3GcJa3g\#v=onepage\&q=Quality $\% 20$ control\%20 methods $\% 20$ for $\% 20$ medicinal $\% 20$ plant $\% 20$ materials $\% 2 \mathrm{C} \& \mathrm{f}=$ false

World Health Organization. (2011). Quality control methods for herbal materials. Recuperado de: https://apps.who.int/iris/bitstream/handle/10665/ 44479/9789241500739_eng.pdf 